\title{
Les lignes directrices de la Société canadienne de thoracologie pour la MPOC : Un résumé des recommendations à l'intention des médecins de famille
}

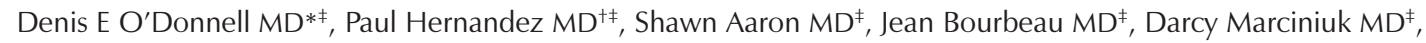 \\ Rick Hodder MD , Meyer Balter MD, Gordon Ford MD, André Gervais MD, Roger Goldstein MD, François Maltais MD, \\ Jeremy Road MD, Valoree McKay ${ }^{\S}$, Jennifer Schenkel ${ }^{\S}$ \\ *Président, comité d'élaboration des lignes directrices de la SCT au sujet de la BPCO; ${ }^{P}$ Président, comité de mise en œuvre \\ et de diffusion; ${ }^{\ddagger}$ Comité de rédaction; \$Personnel administratif de l'Association pulmonaire du Canada
}

\begin{abstract}
I a maladie pulmonaire obstructive chronique (MPOC) est une Lcause importante d'incapacité et de mortalité de par le monde. Le présent projet d'éducation de la Société canadienne de thoracologie (SCT) vise principalement à optimiser le diagnostic précoce, la prévention et la prise en charge de la MPOC au Canada.
\end{abstract}

\section{Lépidémiologie}

La MPOC, qui touche environ $4 \%$ des adultes canadiens, représente une préoccupation majeure en matière économique et de santé. En 1999, la MPOC était la quatrième cause de décès en importance chez les hommes et la cinquième chez les femmes. On prévoit que les taux de morbidité et de mortalité secondaires à la MPOC augmenteront d'ici 15 ans, surtout auprès de la population des femmes vieillissantes.

Message clé 1 : La MPOC est un trouble respiratoire imputable en grande partie au tabagisme, et se caractérise par une obstruction évolutive et partiellement réversible des voies respiratoires, par des manifestations systémiques et par une fréquence et une gravité croissantes des exacerbations. Les effets systémiques comprennent une dysfonction des muscles périphériques, une insuffisance cardiaque droite, une polyglobulie, une altération de l'état nutritionnel et une dépression. Ces facteurs, combinés à une incapacité respiratoire, contribuent à une deterioration de la qualité de vie.

Message clé 2 : Les médecins de famille occupent un rôle de premier plan dans la prise en charge de la MPOC. En qualité de dispensateurs de soins de santé primaires, les médecins de famille sont responsables de dépister et de diagnostiquer précocément la MPOC, d'éduquer les patients, de donner les conseils et assurer un suivi pour favoriser l'abandon du tabac, d'instaurer une pharmacothérapie, de prendre en charge les exacerbations aiguës, d'assurer un suivi à long terme et d'offrir les soins de fin de vie, et les médecins de famille sont dans une position idéale pour assurer les soins et services aux patients avec MPOC.

Message clé 3 : Il est essentiel d'objectiver l'obstruction des voies aériennes par une spirométrie pour poser un diagnostic de MPOC. Un volume expiratoire maximal en une seconde (VEMS) post bronchodilateur inférieur à $80 \%$ de la valeur normale prédite et un ratio entre le VEMS et la capacité vitale forcée inférieur à 0,7 sont tous deux requis pour poser un diagnostic de MPOC.

Message clé 4 : La plupart des patients atteints de MPOC ne sont pas diagnostiqués avant que leur maladie soit très avancée. Une spirométrie, ciblée vers les personnes vulnérables, peux permettre de poser un diagnostic précoce. Les critères suivants sont utiles aux médecins de famille pour cibler les patients de leur pratique à qui faire passer une spirométrie en vue de poser un diagnostic précoce de MPOC :
1. Les fumeurs et les anciens fumeurs de 40 ans et plus;

2. les personnes affichant une toux et des expectorations persistantes; 3. les personnes souffrant de fréquentes infections des voies respiratoires;

4. les personnes présentant un essoufflement évolutif à l'activité physique.

\section{LÉVALUATION DU PATIENT ATTEINT DE MPOC}

L'anamnèse du patient devrait inclure l'histoire du tabagisme avec le nombre de paquets-années, la fréquence et la gravité des exacerbations ainsi qu'une évaluation de la dyspnée et de l'incapacité.

Message clé 5 : L'échelle de dyspnée du Conseil de recherche médicale (figure 1) est une échelle fonctionnelle utile pour évaluer l'essoufflement et l'incapacité, et elle peut contribuer à évaluer la gravité de la maladie (tableau 1). Règle générale, l'examen physique et les radiographies pulmonaires ne permettent pas de poser un diagnostic, mais elles sont utiles pour éliminer les comorbidités et les pathologies associées. La gazométrie du sang artériel devrait être envisagée chez les patients dont le VEMS est inférieur à $40 \%$ de la valeur prévue.

Message clé 6 : La MPOC et l'asthme sont fondamentalement différents, et cette distinction diagnostique devrait être apportée chez chaque patient symptomatique. Il peut être difficile de distinguer la MPOC de l'asthme chez certains patients (p. ex., chez les asthmatiques chroniques qui fument). En cas de MPOC, les symptômes ont tendance à évoluer lentement au fil des ans et à faire

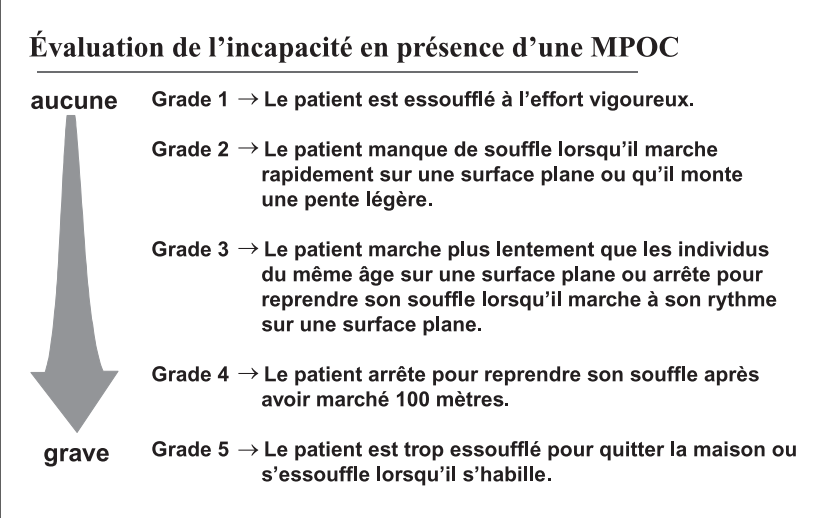

Figure 1) Échelle de dyspnée du Conseil de recherches médicales. MPOC : Maladie pulmonaire obstructive chronique. Données tirées de la référence 1 


\section{TABLEAU 1}

La classification de la maladie pulmonaire obstructive chronique établie par la Société canadienne de thoracologie selon les symptômes/invalidité*

\begin{tabular}{|c|c|}
\hline $\begin{array}{l}\text { Stade de } \\
\text { MPOC }\end{array}$ & Symptômes \\
\hline $\begin{array}{l}\text { À risque } \\
\text { (ne } \\
\text { correspond } \\
\text { pas } \\
\text { "encore » au } \\
\text { diagnostic } \\
\text { de MPOC) }\end{array}$ & $\begin{array}{l}\text { Fumeur asymptomatique, ancien fumeur ou patient } \\
\text { présentant une toux ou des expectorations chroniques, } \\
\text { mais ratio VEMS/capacité vitale forcée } \geq 0,7 \text { et/ou } \\
\text { VEMS } \geq 80 \% \text { de la valeur prédite après l'administration } \\
\text { d'un bronchodilatateur }\end{array}$ \\
\hline Bénigne & $\begin{array}{l}\text { Patient essoufflé en raison de la MPOC } \mathrm{MP}^{\dagger} \text { lorsqu'il est pressé } \\
\text { ou qu'il monte une pente légère (CRM 2) }\end{array}$ \\
\hline Modéré & $\begin{array}{l}\text { Patient essoufflé en raison de la MPOC', ce qui l'oblige à } \\
\text { marcher plus lentement que des individus du même âge sur } \\
\text { une surface plane ou à s'arrêter au bout d'environ } 100 \mathrm{~m} \\
\text { (ou de quelques minutes) sur une surface plane } \\
\text { (CRM 3-4) }\end{array}$ \\
\hline Grave & $\begin{array}{l}\text { Patient essoufflé en raison de la MPOC†, ce qui l'empêche } \\
\text { de quitter la maison ou le rend essoufflé lorsqu'il s'habille } \\
\text { ou se déshabille (CRM 5) ou présence d'une insuffisance } \\
\text { respiratoire chronique ou de signes cliniques } \\
\text { d'insuffisance cardiaque droit }\end{array}$ \\
\hline
\end{tabular}

*Un ratio entre le volume expiratoire maximal en une seconde (VEMS) et la capacité vitale forcée inférieur à 0,7 et un VEMS inférieur à $80 \%$ de la valeur prédite après l'administration d'un bronchodilatateur sont tous deux requis pour poser un diagnostic de MPOC; ${ }^{\dagger} E n$ présence de pathologies non reliées à la MPOC et susceptibles de provoquer un essoufflement (p. ex., dysfonction cardiaque, anémie, faiblesse musculaire, troubles métaboliques), les symptômes ne reflètent pas nécessairement la gravité de la MPOC. La classification de la gravité de la MPOC devrait être effectuée avec prudence chez les patients atteints de maladies comorbides ou d'autres éléments contribuant à l'essoufflement. CRM : Conseil de recherche médicale

leur apparition chez des patients plus âgés qui ont d'importants antécédents de tabagisme. Les patients atteints de MPOC ne normalisent jamais leur fonction pulmonaire. Il est risqué de confondre l'asthme et la MPOC, car cette confusion peut favoriser l'usage inopportun de corticostéroïdes en inhalation chez des patients atteints de MPOC ou retarder l'usage de corticostéroïdes inhalés chez les patients asthmatiques.

Message clé 7 : Référer le patient à un spécialiste dans les cas suivants :

1. Le diagnostic est incertain.

2. Les symptômes sont graves ou disproportionnés par rapport à la gravité de l'obstruction des voies aériennes tel que mesuré par la spirométrie.

3. Les symptômes font leur apparition chez un patient plus jeune (moins de 40 ans).

Les spécialistes peuvent vous assister dans la prise en charge de la MPOC chez les patients qui ne répondent pas à une combinaison de bronchodilatateurs, qui présentent des exacerbations graves ou récurrentes, qui souffrent de comorbidités complexes, qui ont besoin d'une réadaptation pulmonaire, qui doivent se faire évaluer pour entreprendre une oxygénothérapie à domicile ou qui pourraient être candidats à une intervention chirurgicale (p. ex., transplantation pulmonaire, chirurgie de l'emphysème ou bullectomie).

\section{LA PRISE EN CHARGE DE LA MPOC}

Message clé 8 : La MPOC est une maladie traitable mais non curable. Une stratégie de prise en charge incluant une pharmacothérapie et des interventions non pharmaceutiques peut réduire les symptômes, accrôtre la capacité d'exercice et la qualité de vie avec efficacité, même chez les patients atteints d'une MPOC grave. La prise en charge de la MPOC vise à prévenir la progression de la maladie, à soulager les symptômes, à améliorer la tolérance a l'exercice, à prévenir et à traiter les exacerbations à améliorer la qualité de vie, et si possible réduire le taux de mortalité. Pour ce faire, il faut adopter une ligne de conduite progressive selon laquelle le traitement s'intensifie compte tenu de la gravité des symptômes et de l'incapacité (figure 2).

Message clé 9 : L'abandon du tabac représente la seule intervention pouvant réduire le risque d'apparition d'une MPOC, et la seule intervention pouvant en ralentir l'évolution. Au moins $70 \%$ des fumeurs consultent un médecin chaque année, et les conseils promulguer par un médecin sont cités parmi les éléments importants qui motivent les fumeurs à arrêter de fumer. Chaque fumeur devrait recevoir au moins un court message. Toutefois, dans la mesure du possible, un traitement plus intense (thérapie individuelle et de groupe, adoption d'un traitement de substitution de la nicotine ou administration de bupropion), qui procure un taux d'abandon plus élevé, devrait être privilégié.

Message clé 10 : L'éducation du patient et de sa famille, associée à une supervision et à un soutien fondés sur des principes d'auto-prise en charge propres à la maladie, est précieuse. En plus d'améliorer les habiletés d'adaptation et la qualité de vie, les programmes d'auto-prise en charge réduisent le nombre d'hospitalisations et les coûts reliés aux soins de santé. La collaboration entre le patient, sa famille et les éducateurs en MPOC bien formés est essentielle au succès des programmes d'éducation et d'auto-prise en charge.

Message clé 11 : Les vaccins préviennent les exacerbations de la MPOC. Les patients atteints de MPOC ayant la grippe sont plus vulnérables aux pneumopathies nécessitant des hospitalisations et augmentant la mortalité. C'est pourquoi l'administration du vaccin contre l'influenza est recommandée tous les ans. Les bénéfices du vaccin antipneumococcique sont moins bien établis, mais il est recommandé de l'administrer au moins une fois à tous les patients atteints de MPOC, et peut-être tous les cinq à dix ans.

Message clé 12 : Les bronchodilatateurs sont le pilier de la pharmacothérapie de la MPOC. Ils peuvent réduire la rétention d'air (hyperinflation pulmonaire) et la dyspnée et améliorer la qualité de vie même si la spirométrie ne démontre aucune amélioration (figure 3).

- Chez les patients qui présentent un essoufflement à l'effort et une incapacité minime, des béta 2 -agonistes à courte durée d'action (ou un anticholinergique régulier ou une association d'anticholinergique et de béta ${ }_{2}$-agoniste), administrés au besoin, devraient constituer le traitement initial. Le choix du traitement de première intention dépend de la réponse clinique et des effets secondaires.

- Si les symptômes sont persistants, l'ajout d'un bronchodilatateur à action prolongée, tel qu'un anticholinergique (tiotropium $18 \mu \mathrm{g}$ qd) ou un béta ${ }_{2}$-agoniste à longue durée d'action (BALA) $(12 \mu \mathrm{g}$ de formotérol bid ou $50 \mu \mathrm{g}$ de salmétérol bid), est recommandé. L'utilisation d'un bronchodilatateur à courte durée d'action est maintenue, au besoin, afin de favoriser un soulagement immédiat des symptômes.

- Chez les patients qui présentent des symptômes persistants modérés à graves, une association d'anticholinergique à action prolongée et de BALA est recommandée en plus d'un béta 2 agoniste à courte durée d'action, au besoin, afin de favoriser un soulagement immédiat des symptômes.

- Chez les patients qui présentent des symptômes graves malgré un anticholinergique à action prolongée et un BALA, il est possible de faire l'essai de la théophylline orale à action prolongée. Il est alors nécessaire de surveiller le taux de théophylline afin de 


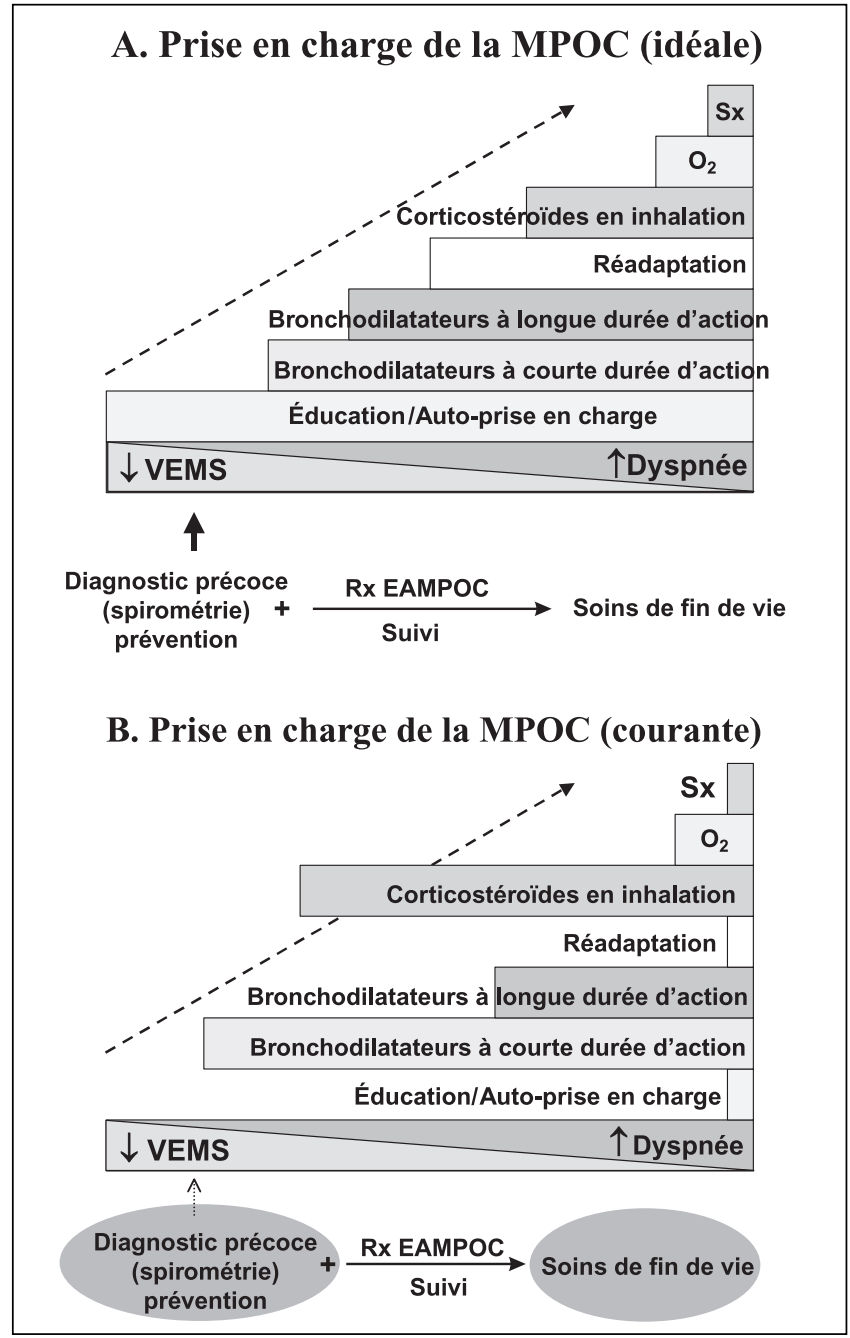

Figure 2) A Paradigme de prise en charge progressive de la maladie pulmonaire obstructive chronique (MPOC) d'après l'accroissement des symptômes et de l'incapacité. B Les carences actuelles de la prise en charge incluent l'absence de spirométrie pour un diagnostic précoce, d'éducation et de réadaptation, la surutilisation des corticostéroïdes en inhalation au début de la maladie et l'absence de soins de fin de vie structurés. VEMS : Volume expiratoire maximal en une seconde; $\mathrm{O}_{2}$ : Oxygénothérapie; $\mathrm{Rx}$ EAMPOC : Traitement des exacerbations aiguës de la MPOC; Sx : Intervention de l'emphysème ou transplantation pulmonaire

prévenir ou minimiser les effets secondaires ou les interactions avec d'autres médicaments.

- Un traitement d'entretien prolongé aux corticostéroïdes oraux ne procure aucun bénéfice démontré dans les cas de MPOC, et il s'associe à un risque élevé d'effets secondaires systémiques.

- Contrairement au traitement de l'asthme, les corticostéroïdes en inhalation (CSI) ne devraient pas être utilisés en guise de traitement de première intention de la MPOC. Cependant, les CSI devraient être envisagés chez les patients atteints d'une MPOC modérée à grave qui souffrent d'au moins trois exacerbations aiguës par année, surtout si celles-ci exigent une corticothérapie orale.

- Les patients qui demeurent essoufflés malgré un traitement optimal aux bronchodilatateurs à longue durée d'action pourraient bénéficier d'une association de CSI et de BALA, mais cette possibilité devrait être évaluée en fonction de chaque individu.

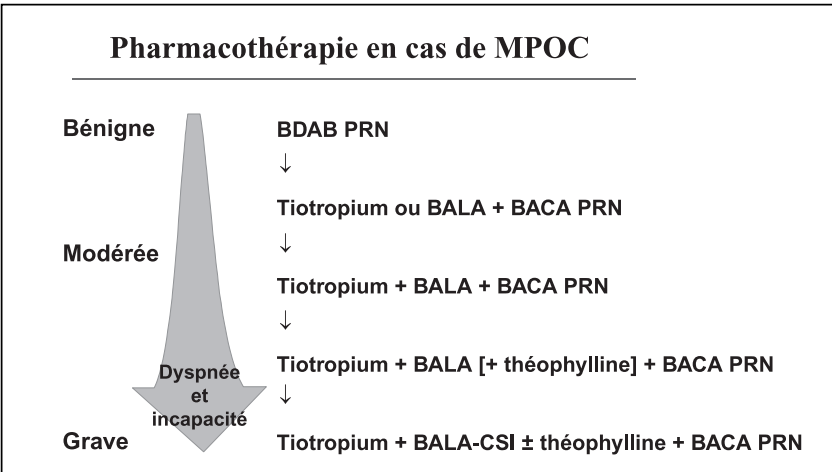

Figure 3) Pharmacothérapie de la maladie pulmonaire obstructive chronique (MPOC) selon l'accroissement des symptômes et de l'incapacité. [+ théophylline] : Ajouter si elle est tolérée; BACA Béta ${ }_{2}$-agoniste à courte durée d'action (c.-à.-d., salbtamol); BALA : Béta_-agoniste à longue durée d'action (c.-à.-d., formotérol ou salmétérol); BALA-CSI : Préparation de BALA associé à des corticostéroüdes en inhalation; $\mathrm{BDAB}:$ Bronchodilatateur à courte durée d'action (beta ${ }_{2}$-agonistes ou anticholinergiques)

Message clé 13 : Tous les patients atteints de MPOC devraient être encouragés à conserver un mode de vie actif. Les patients atteints de MPOC qui présentent un essoufflement à l'activité ont tendance à réduire leur niveau d'activité afin d'éviter de déclencher un inconfort respiratoire. Un déconditionnement secondaire à l'inactivité contribue à une dysfonction des muscles squelettiques, au point que même une activité mineure provoque une fatigue. Un programme d'exercice est recommandé pour les patients atteints de MPOC qui sont cliniquement stables et qui demeurent essoufflés et limités dans leurs activités malgré l'utilisation optimale de bronchodilatateurs. Il est démontré que les programmes de réadaptation pulmonaire qui incluent des exercices supervisés et l'éducation des patients réduisent considérablement l'essoufflement, accroissent l'endurance à l'exercice et la qualité de vie et peuvent réduire le nombre et la durée des hospitalisations chez les patients atteints de MPOC.

Message clé 14 : Les exacerbations aiguës de MPOC (EAMPOC) constituent la principale cause de consultation médicale, d'hospitalisation et de décès chez les patients atteints de MPOC. L'EAMPOC se définit comme une aggravation soutenue de la dyspnée, de la toux ou des expectorations, qui entraîne un recours accru ou l'ajout de médicaments. De plus, l'EAMPOC se classe comme étant purulente ou non purulente. L'antibiothérapie devrait être envisagée chez les patients atteints d'EAMPOC purulente. Une anamnèse, un examen physique et des radiographies pulmonaires sont recommandés chez les patients atteints d'EAMPOC. Une culture des expectorations devrait être envisagée chez les patients dont la fonction pulmonaire est très mauvaise, qui présentent de fréquentes exacerbations ou qui ont été sous antibiothérapie au cours des trois mois précédents. Une spirométrie devrait être effectuée chez les patients présumés atteints d'une MPOC, mais seulement après leur rétablissement et lorsque leur état s'est stabilisé.

- Il faudrait utiliser une thérapie d'association aux béta 2 -agonistes à courte durée d'action et aux bronchodilatateurs

anticholinergiques pour traiter la dyspnée en présence d'une EAMPOC. Les patients qui prennent déjà une méthylxanthine orale peuvent poursuivre leur traitement pendant une EAMPOC, mais ce médicament n'a aucun rôle à jouer s'il s'agit d'une thérapie initiale. 
TABLEAU 2

Recommandations en matière d'antibiothérapie dans les cas d'exacerbations purulentes aiguës d'une maladie pulmonaire chronique obstructive (MPOC)

\begin{tabular}{|c|c|c|c|c|c|}
\hline Groupe & État clinique & $\begin{array}{l}\text { Symptômes et } \\
\text { facteurs de risque }\end{array}$ & Pathogènes probables & Premier choix & $\begin{array}{l}\text { Choix } \\
\text { alternatives }\end{array}$ \\
\hline Simple & $\begin{array}{l}\text { MPOC sans } \\
\text { facteur de } \\
\text { risque }\end{array}$ & $\begin{array}{l}\text { Accroissement de la toux et des } \\
\text { expectorations, expectorations } \\
\text { purulentes et accroissement de } \\
\text { la dyspnée }\end{array}$ & $\begin{array}{l}\text { Haemophilus influenzae, } \\
\text { Moraxella catarrhalis, } \\
\text { Streptococcus pneumoniae }\end{array}$ & $\begin{array}{l}\text { Amoxicilline, doxycycline, } \\
\text { triméthoprime- sulfaméthoxazole, } \\
\text { céphalosporines de } 2^{\mathrm{e}} \text { et } \\
3^{\mathrm{e}} \text { génération, } \\
\text { macrolides à large spectre }\end{array}$ & $\begin{array}{l}\text { Inhibiteurs de la } \\
\text { béta-lactamine ou } \\
\text { de la } \\
\text { béta-lactamase, } \\
\text { fluoroquinolones }\end{array}$ \\
\hline $\begin{array}{l}\text { Complexe } \\
\text { des facteurs }\end{array}$ & $\begin{array}{l}\text { MPOC associée à } \\
\text { de } \\
\text { risque }\end{array}$ & $\begin{array}{l}\text { Identique à l'exacerbation } \\
\text { simple, et au moins l'un } \\
\text { des éléments suivants: } \\
\text { - VEMS < } 50 \text { \% de la valeur prédite } \\
\text { - } \geq 4 \text { exacerbations/année } \\
\text { - Cardiopathie ischémique } \\
\text { - Oxygénothérapie à domicile } \\
\text { - Corticothérapie orale chronique } \\
\text { - Antibiothérapie au cours des trois } \\
\text { mois précédents }\end{array}$ & $\begin{array}{l}\text { Identique à l'exacerbation } \\
\text { simple, plus: } \\
\text { - Klebsiella et autres (culture } \\
\text { gram négatif) } \\
\text { - Probabilité accrue de résistance } \\
\text { aux béta-lactamines }\end{array}$ & $\begin{array}{l}\text { Inhibiteur de la béta-lactamine ou } \\
\text { de la béta-lactamase; } \\
\text { fluoroquinolones (antibiotiques } \\
\text { pour patients sans complication } \\
\text { mais sur stéroïdes oraux) }\end{array}$ & $\begin{array}{l}\text { Peut nécessiter } \\
\text { une thérapie iv } \\
\text { considérer } \\
\text { une référence } \\
\text { à un spécialiste }\end{array}$ \\
\hline
\end{tabular}

VEMS : volume expiratoire maximal en une seconde

- Une corticothérapie orale ou intraveineuse devrait être administrée pendant 14 jours à la plupart des patients atteints d'une MPOC modérée à grave présentant une EAMPOC. Cependant, des périodes de traitement plus courtes, de sept à 14 jours, peuvent également être efficaces. Des doses quotidiennes de $25 \mathrm{mg}$ à $50 \mathrm{mg}$ de prednisone sont recommandées.

- Les antibiotiques sont bénéfiques en cas d'EAMPOC grave, qui se traduit par des épisodes associés à un accroissement de la dyspnée et de la purulence ou du volume des expectorations. Chez les patients avec une EAMPOC simple (tableau 2) ne présentant aucun facteur de risque les antibiotiques relativement peu coûteux peuvent alors cibler les pathogènes probables. Les patients atteints d'une EAMPOC complexe présentent des facteurs de risque peuvent être infecter par des organismes plus virulents ou résistants. Si un patient a besoin de répéter l'antibiothérapie dans un délai de trois mois, une nouvelle classe d'antibiotiques devrait être utilisée afin de réduire le risque de résistance au minimum.
- Dans les cas d'EAMPOC graves compliquées par une insuffisance respiratoire aiguë qui ne répond pas à un traitement initial aux bronchodilatateurs, un soutien ventilatoire par méthode non invasive peut être indiqué et bénéfique. Une consultation auprès d'un spécialiste est alors recommandée.

Message clé 15 : La MPOC est une maladie débilitante qui évolue vers l'insuffisance respiratoire et le décès. Les médecins ont aussi la responsabilité de soutenir les patients atteints de MPOC et leurs soignants en fin de vie.

Veuillez vous reporter au supplément A du volume $10 \mathrm{du}$ Canadian Respiratory Journal pour obtenir le résumé de l'article. L'intégralité des lignes directrices de la SCT pour la MPOC sera publiée à une date ultérieure.

\section{RÉFÉRENCE}

1. Fletcher CM, Elmes PC, Wood CH. The significance of respiratory symptoms and diagnosis of chronic bronchitis in a working population. Br Med J 1959;1:257-66. 


\section{ERratum}

O’Donnell DE, Hernandez P, Aaron S, et al. Les lignes directrices de la Société canadienne de thoracologie pour la MPOC : Un résumé des recommendations à l'intention des médecins de famille. Can Resp J 2003;10(6):316-319.

Due to translation errors, the above guidelines have been retranslated and republished in full in this issue of the Canadian Respiratory Journal (pages 463-466). We apologize for any inconvenience this may have caused. 


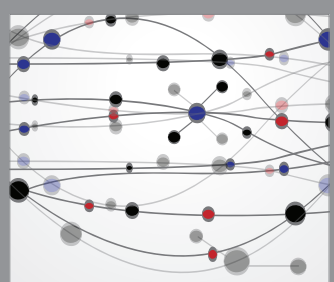

The Scientific World Journal
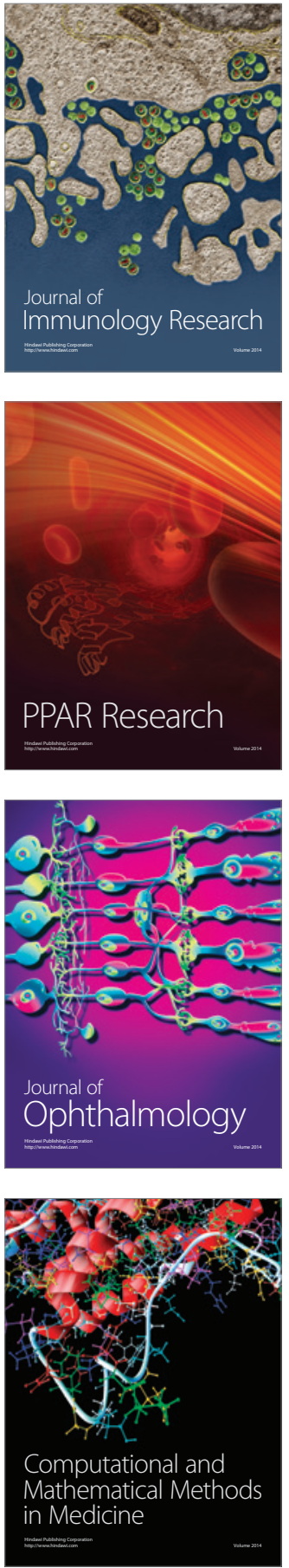

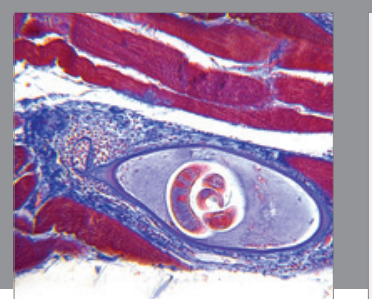

Gastroenterology Research and Practice

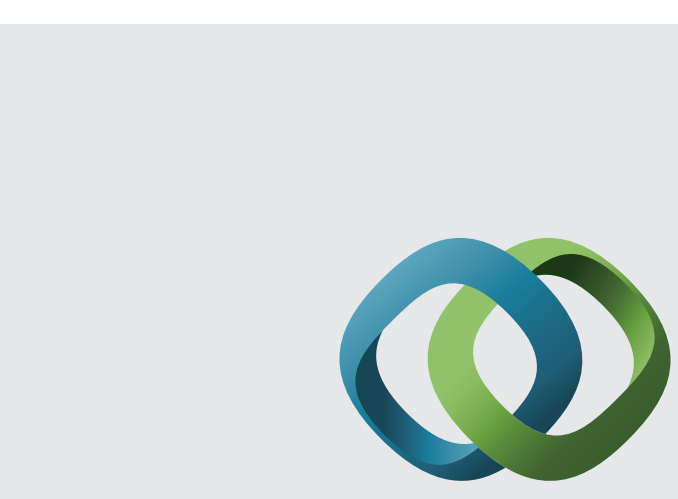

\section{Hindawi}

Submit your manuscripts at

http://www.hindawi.com
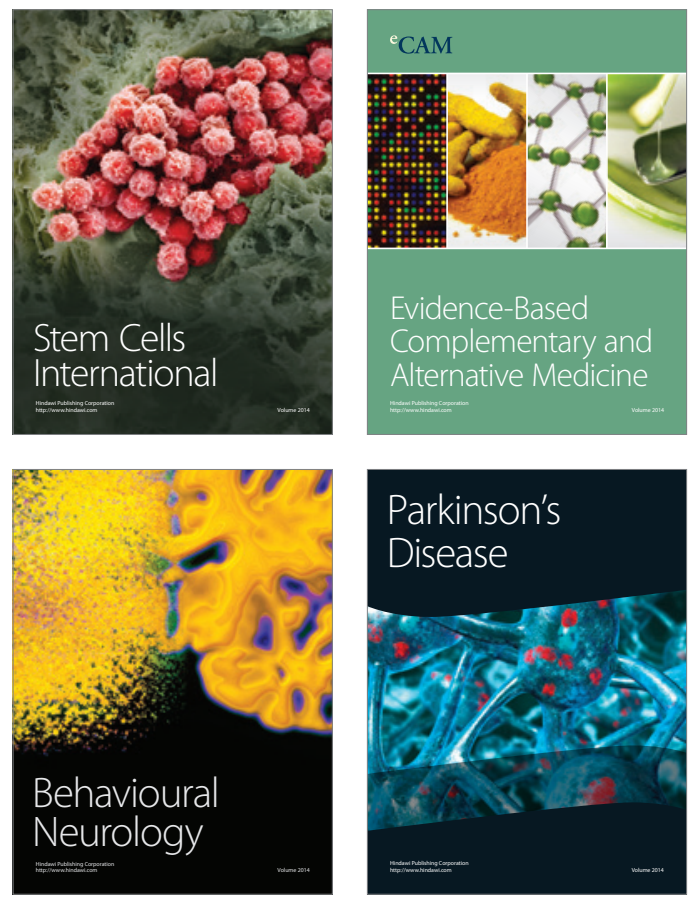
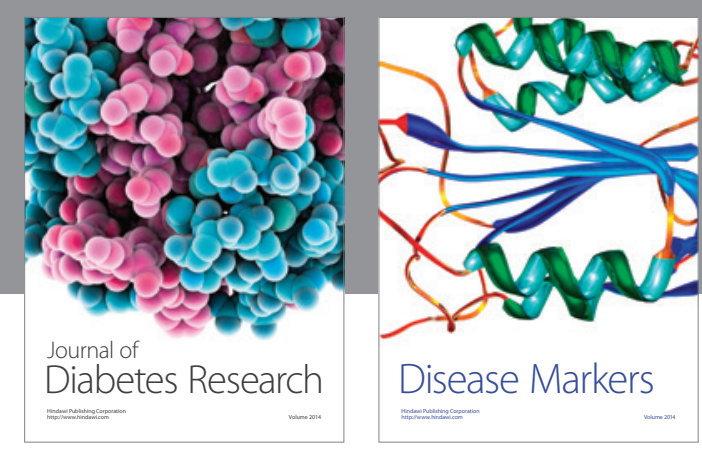

Disease Markers
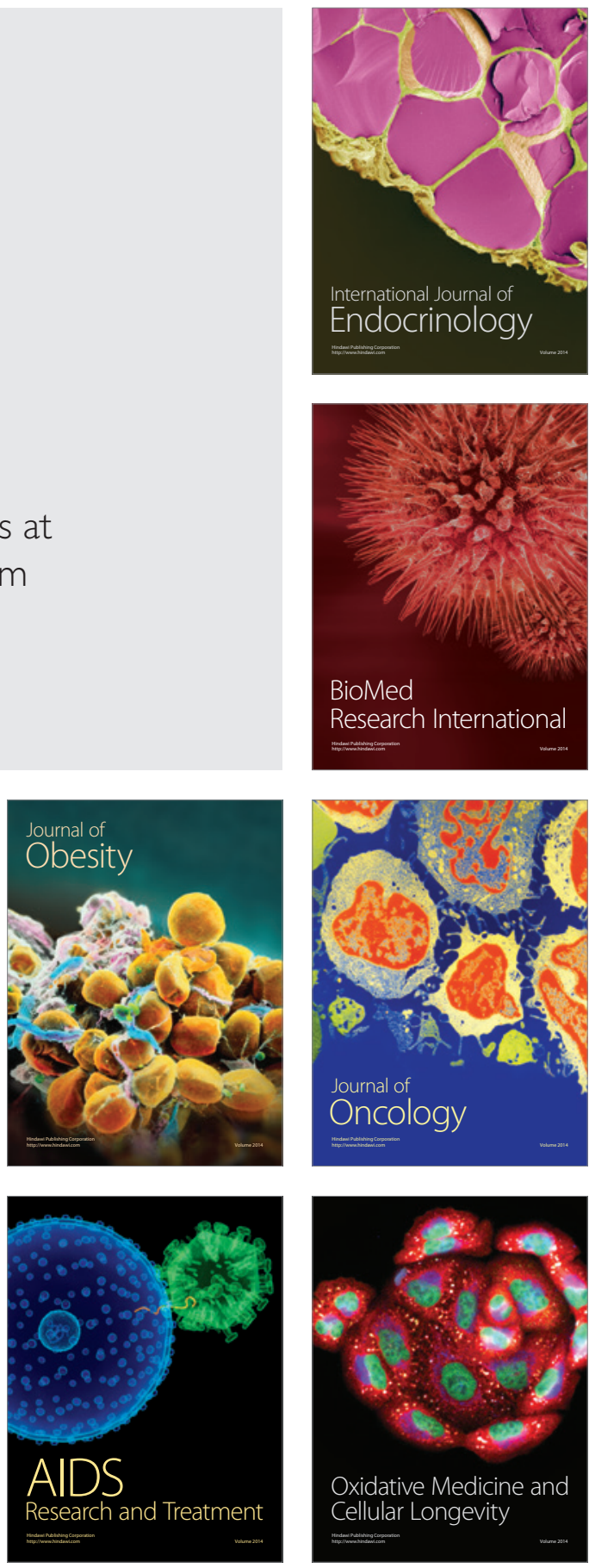